-研究报告・

\title{
轻小型无人机航摄技术辅助的热带森林 样地测量精度问题探讨
}

\author{
邓 云1,3,4* 王 涁 ${ }^{2}$ 李 强 ${ }^{2}$ 张志明 2 \\ 邓晓保 ${ }^{1,3}$ 曹 敏 ${ }^{1}$ 林露湘 ${ }^{1,3}$ \\ 1 (中国科学院西双版纳热带植物园热带森林生态学重点实验室, 云南预腊 666303) \\ 2 (云南大学生态学与环境科学学院, 昆明 650091) \\ 3 (中国科学院西双版纳热带植物园云南西双版纳森林生态系统国家野外科学观测研究站, 云南预腊 666303) \\ 4 (中国科学院大学, 北京 100049)
}

摘要: 准确的样地坐标位置是无人机航摄数据与地面调查数据融合使用的必要前提, 但是在森林样地的具体实践 中, 会有许多因素制约着样地位置的测量精度, 这有可能影响后期的数据融合过程甚至得出错误的结论, 研究者 们需要对此予以足够的重视。本文通过对比西双版纳地区 10 个热带森林样地及周围区域无人机航摄过程中的地面 控制点测量精度、Photoscan摄影测量软件所得点云解算精度和照片曝光点重投影精度, 发现: (1)即使使用性能相 对较好的实时差分(real time kinematic, RTK)式GNSS系统进行定位, 在林内也很难获得很好的定位精度, 林窗处 的地面控制点均方根误差(root mean square error, RMSE) 在水平和垂直方向分别为 $0.167 \pm 0.158 \mathrm{~m}$ 和 $0.297 \pm 0.170 \mathrm{~m}$, 林下样地顶点桩处分别为 $0.392 \pm 0.368 \mathrm{~m}$ 和 $0.657 \pm 0.412 \mathrm{~m}$; (2)软件的全局解算精度主要受控制点地面测量精度 和控制点数量的影响; (3)若仅依托普通的单站式GPS对无人机位置进行定位, 则照片曝光点的重投影坐标位置可 能存在较大误差(RMSE在水平和垂直方向上分别为 $18.434 \pm 5.252 \mathrm{~m}$ 和34.042 $\pm 6.920 \mathrm{~m}$ ); (4)估测地形与实测地形 间的高差标准差与林冠平均高度正相关 $(r=0.713, P<0.05)$, 估测地形模型在 20 ha样地尺度下的验证结果优于 1 ha样地。基于以上结果, 我们建议: (1)在对热带森林进行无人机航摄的过程中, 必须有足够数量和质量的分布相对 均匀的地面控制点对测量误差进行控制; (2)摄影测量法的优势在于能够以相对简单的前端设备建立数字表面模 型, 但该方法可能很难在森林样地中建立准确的数字地形模型。在使用无人机获取数据之前, 研究者应预先考虑 到适合自己的恰当方法以应对以上的精度控制问题。

关键词: 轻小型无人机; 全球导航卫星系统; 定位精度; 森林样地

\section{An analysis of lightweight-drone-assisted mapping accuracy in tropical forest plot}

Yun Deng ${ }^{1,3,4^{*}}$, Bin Wang ${ }^{2}$, Qiang $\mathrm{Li}^{2}$, Zhiming Zhang ${ }^{2}$, Xiaobao Deng ${ }^{1,3}$, Min Cao ${ }^{1}$, Luxiang Lin $^{1,3}$

1 CAS Key Laboratory of Tropical Forest Ecology, Xishuangbanna Tropical Botanical Garden, Chinese Academy of Sciences, Mengla, Yunnan 666303

2 School of Ecology and Environmental Sciences, Yunnan University, Kunming 650091

3 National Forest Ecosystem Research Station at Xishuangbanna, Xishuangbanna Tropical Botanical Garden, Chinese Academy of Sciences, Mengla, Yunnan 666303

4 University of Chinese Academy of Sciences, Beijing 100049

Abstract: Accurate coordinate position is a prerequisite for combining drone-assisted remotely sensed data and ground survey data. However, in the practice of surveying forests, many factors prevent accurate measurement of coordinate position and inaccurate coordinates may lead to incorrect conclusions. Therefore, researchers must pay attention to factors effecting accuracy of position. In this study, we compared location error of ground

收稿日期: 2018-02-06; 接受日期: 2018-08-05

基金项目: 国家重点研发专项(2016YFC0500202)

* 通讯作者 Author for correspondence. E-mail: dy@xtbg.org.cn 
control points (GCPs), model error of photogrammetric point cloud (estimated by Photoscan software) and reprojection error of camera exposure position. First, we found that real time kinematic (RTK) global navigation satellite system (GNSS) cannot locate position in tropical forest with high accuracy. The root mean square error (RMSE) of GCPs in canopy gaps were $0.167 \pm 0.158 \mathrm{~m}$ and $0.297 \pm 0.170 \mathrm{~m}$ in the horizontal and vertical axes respectively. In comparison, RMSE of GCPs within forests were $0.392 \pm 0.368 \mathrm{~m}$ and $0.657 \pm 0.412 \mathrm{~m}$ respectively for horizontal and vertical axes. Second, the number and measurement accuracy of GCPs influenced model error of photogrammetric point cloud. Third, reprojection error of camera exposure position (18.434 $\pm 5.252 \mathrm{~m}$ and $34.042 \pm 6.920 \mathrm{~m}$ in horizontal and vertical axes respectively) was much greater than location error of GCPs when the drone acquired position with a single-station GPS system.

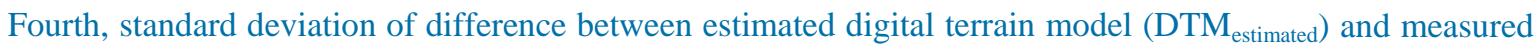
digital terrain model $\left(\mathrm{DTM}_{\text {measured }}\right)$ was positively correlated with mean canopy height $(r=0.713, P<0.05)$. DTM $_{\text {estimated }}$ was better estimated at 20 ha scale than at 1 ha scale. Based on these results, we suggest that uniform distribution and sufficient numbers of GCPs can improve drone-assisted mapping accuracy. Lightweight-drone-based photogrammetry has an advantage in requiring fewer equipment and enabling creation of accurate DSM (digital surface model), but remains incapable of estimating ground elevation. Researchers should consider these factors related to accuracy before using drones for surveys.

Key words: lightweight drone; global navigation satellite system; location accuracy; forest plot

近年来基于无人机的近地面遥感技术正被广 泛应用于生态学研究之中, 在生物多样性保护(Koh \& Wich, 2012)、野生动物监测(Christie et al, 2016)、 植被分布调查(Husson et al, 2016)、森林生物量测算 (Cunliffe et al, 2016)、灾害监测(李云等, 2011)、森 林防火(马瑞升等, 2008)、森林资源调查(张园等, 2011)等许多领域都得到使用和推广。相对于有人机 和大中型无人机, 我国目前对轻小型无人机使用上 的管理相对宽松。根据正在征求意见的《无人驾驶 航空器飞行管理暂行条例》中的相关规定(中国民用 航空局, 2018), 空机质量0.25-4 kg (或最大起飞重 量不超过 $7 \mathrm{~kg}$ ) 的轻型无人机和空机质量 $4-15 \mathrm{~kg}$ (或 最大起飞重量不超过 $25 \mathrm{~kg}$ ) 的小型无人机只需完成 产品认证而不必做适航管理, 且轻型无人机在适飞 空域上方不超过飞行安全高度飞行, 或是具备可靠 被监视和空域保持能力的小型无人机在轻型无人 机适飞空域及上方不超过飞行安全高度飞行时, 可 不单独划设隔离空域运行。对于森林生态学研究的 样地定位监测工作而言, 考虑到日常监测的时间频 度较高, 监测区域地形复杂, 以及一线监测人员常 不具备专业飞手资格等因素, 政策管理相对宽松且 使用相对简单的轻小型无人机可能更适用于生态 学的日常的监测和科研活动。

作为新型的遥感平台, 无人机平台相对传统机 载和星载平台的最大优势在于其获取的数据能够 有更高的空间分辨率, 但是, 若希望将之与地面实
际调查数据进行匹配与融合分析, 还需要分别对基 于空中和地面平台所采集的数据的坐标进行精确 匹配。虽然近年来结构动态重建(structure from motion, SfM) 算法在摄影测量技术中正逐渐普及, 许多软件已能够将轻小型无人机获取的照片直接 用于正射影像和数字表面模型的生产(Westoby et al, 2012), 但是, 以往分辨率为 $10 \mathrm{~m}$ 左右的卫星影像依 托普通的米级精度的手持定位终端即可满足误差 控制的需要, 而当使用无人机作为遥感平台时, 影 像分辨率通常会高达厘米级, 此时若不对测量误差 进行小心控制, 则很有可能产生不同平台采集的数 据坐标无法严格匹配的现象, 进而导致不同来源数 据间的融合结果不理想, 甚至得出错误的结论。

对无人机获取影像的摄影测量处理时, 首先需 要以一定数量的已知准确坐标的地面控制点作为 绝对定向的基础, 在此之上进行影像空三加密 ${ }^{1}$, 依据摄影测量过程的几何反转, 重建出可以量测的 几何立体模型(张剑清等, 2003)。在此过程中, 地面 控制点 (ground control points, GCPs)或称像控点 (photo control points)作为影像处理所必需的基础资 料, 其数量、精度和分布坐标的准确与否对最终的 数据精度评估至关重要(Harwin \& Lucieer, 2012), 会直接影响树高、冠幅等进阶信息提取的准确性。 由于目前森林生态学研究中无人机用户的学科背

(1) 空三加密, 即解析空中三角测量, 是根据少量已知坐标的地面控制 点, 按照一定的数学模型, 解算出待定点(或加密点)的高程和平面位置 的过程。 
景多以生态学和林学为主, 同时具备测绘专业背景 知识的人员较少, 一些坐标测量过程中的误差程度 及其来源可能会被无意忽视, 因此, 本研究试图通 过对若干无人机航摄技术辅助下的森林样地定位 案例中的测量精度进行对比, 评估森林样地地面定 位作业时的误差控制对无人机航测精度的影响，以 此强调生态学研究中地面坐标测量时的误差控制 对不同获取来源的数据间融合比较的重要性, 为基 于轻小型无人机平台的近地面遥感工作提供误差 控制方面的经验参考。

\section{1 研究区概况}

本研究所涉及的全部样地均位于云南省西双 版纳傣族自治州(以下简称西双版纳), 具体分布见 图1。西双版纳位于我国云南省最南端, 气候属于西 部型季风气候, 年温差小, 日温差大, 干、湿季节变 化特别明显。本区大部分地区为具有亚热带至温带 气候的热带山地, 典型热带气候地区主要集中在海 拔900-1,000 m以下的低山、河谷及坝区，约占该地 区国土总面积的18\%。本地区的植物区系属于热带 亚洲区系的一部分，带有明显热带边缘性质和几种 地理成分交汇的特点, 与广西热带植物区系和海南 热带植物区系在发生上同源, 主要来自于古南大陆 与古北大陆区系成分的融合, 在发展上受热带亚

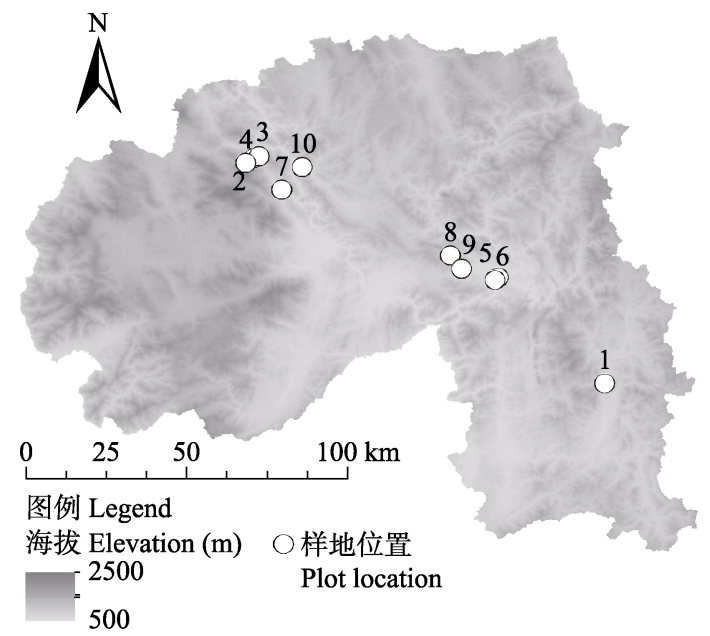

图1 研究样地分布位置。1: 补蚌; 2: 过门山; 3: 茶地头; 4: 大平掌; 5: 石灰山; 6: 新开地; 7: 茶厂后山; 8: 44公里; 9: 蛙仑水库; 10: 江边站。

Fig. 1 Distribution of sample plots. 1, Bubeng; 2, Guomenshan; 3, Chaditou; 4, Dapingzhang; 5, Shihuishan; 6, Xinkaidi; 7, Chachanghoushan; 8, 44 Gongli; 9, Menglunshuiku; 10, Jiangbianzhan.
洲植物区系的渗透和强烈影响(朱华等, 2001)。

\section{研究方法}

\section{1 地面控制点布标与测量}

本研究所用的 2 个 20 ha 样地和 8 个 1 ha样地建 设于2007-2017年间，样地基本情况见表1，所有样 地均位于当地代表性的热带森林地段上。2017年 4-5月，我们在各样地周围布设地面控制点(图2)以 辅助航测数据处理(图3), 每样地航测区域不少于5 个。地面控制点测量时使用X91实时差分式(real time kinematic, RTK) GNSS系统(上海华测导航技术 股份有限公司，上海，中国)，以两脚撑杆对对中杆 进行固定，在设备的控制点测量模式下进行记录， 即以 1 次/ $\mathrm{s}$ 的频度在每个测点上连续记录 $10 \mathrm{~s}$, 取平 均值作为该坐标, 并计算该点测量结果的均方根误 差(root mean square error, RMSE)。

本研究中使用UTM zone 47N坐标系, 通过均 方根误差对数据点的误差情况进行描述(Tomastik et al, 2017)。计算公式如下:

$$
\begin{aligned}
& \operatorname{RMSE}_{x}=\sqrt{\frac{\sum_{i=1}^{n} \Delta x_{i}^{2}}{n}} \\
& R M S E_{y}=\sqrt{\frac{\sum_{i=1}^{n} \Delta y_{i}^{2}}{n}} \\
& \operatorname{RMSE}_{z}=\sqrt{\frac{\sum_{i=1}^{n} \Delta z_{i}^{2}}{n}}
\end{aligned}
$$

式中, $\Delta x_{i} 、 \Delta y_{i} 、 \Delta z_{i}$ 分别是 $x$ (东西)、y(南北)、 $z$ (海拔 $)$ 方向上实测坐标与软件生成的点云坐标间的差值, $n$ 是测量点的数量。由于测量点的水平精度对于精 度评价而言有着重要意义, 因此通过下式单独计算 水平方向上的均方根误差 $R M S E_{x y}$ :

$$
R M S E_{x y}=\sqrt{R M S E_{x}^{2}+R M S E_{y}^{2}}
$$

全局误差 $R M S E_{\text {total }}$ 由以下公式计算:

$$
R M S E_{\text {total }}=\sqrt{R M S E_{x}^{2}+R M S E_{y}^{2}+R M S E_{z}^{2}}
$$

\section{2 无人机影像获取}

本研究共使用两种机型：机型一为Tarot T960 (温州飞越航模有限公司，温州，中国)配双子星飞 控(零度智控(北京)智能科技有限公司, 北京, 中国), 挂载NEX 5T相机(索尼株式会社，东京，日本)在 
表1 样地基本情况

Table 1 Basic information of sample plots

\begin{tabular}{|c|c|c|c|c|c|c|c|c|c|}
\hline $\begin{array}{l}\text { 序号 } \\
\text { No. }\end{array}$ & $\begin{array}{l}\text { 样地名称 } \\
\text { Plot name }\end{array}$ & $\begin{array}{l}\text { 植被类型 } \\
\text { Vegetation type }\end{array}$ & $\begin{array}{l}\text { 北纬 } \\
\text { North } \\
\text { latitude }\end{array}$ & $\begin{array}{l}\text { 东经 } \\
\text { East } \\
\text { longitude }\end{array}$ & $\begin{array}{l}\text { 海拔 } \\
\text { Elevation } \\
\text { (m) }\end{array}$ & $\begin{array}{l}\text { 飞行高度 } \\
\text { Flying altitude } \\
\text { (m) }\end{array}$ & $\begin{array}{l}\text { 地面分辨率 } \\
\text { e Ground } \\
\text { resolution } \\
\text { (cm/pix) }\end{array}$ & $\begin{array}{l}\text { 航测面积 } \\
\text { Coverage } \\
\text { area }\left(\mathrm{km}^{2}\right)\end{array}$ & $\begin{array}{l}\text { 点云密度 } \\
\text { Point cloud } \\
\text { density } \\
\text { (points } / \mathrm{m}^{2} \text { ) }\end{array}$ \\
\hline 1 & $\begin{array}{l}\text { 补蚌 } \\
\text { Bubeng }\end{array}$ & $\begin{array}{l}\text { 季节雨林 } \\
\text { Seasonal rainforest }\end{array}$ & $21.613^{\circ}$ & $101.580^{\circ}$ & 730 & 362 & 5.52 & 1.85 & 82 \\
\hline 2 & $\begin{array}{l}\text { 过门山 } \\
\text { Guomenshan }\end{array}$ & $\begin{array}{l}\text { 山地雨林 } \\
\text { Montane rainforest }\end{array}$ & $22.246^{\circ}$ & $100.599^{\circ}$ & 1,120 & 300 & 4.47 & 1.87 & 31 \\
\hline 3 & $\begin{array}{l}\text { 茶地头 } \\
\text { Chaditou }\end{array}$ & $\begin{array}{l}\text { 常绿阔叶林 } \\
\text { Evergreen } \\
\text { broad-leaved forest }\end{array}$ & $22.250^{\circ}$ & $100.612^{\circ}$ & 1,284 & 310 & 4.18 & 0.58 & 143 \\
\hline 4 & $\begin{array}{l}\text { 大平掌 } \\
\text { Dapingzhang }\end{array}$ & $\begin{array}{l}\text { 常绿阔叶林 } \\
\text { Evergreen broad- } \\
\text { leaved forest }\end{array}$ & $22.230^{\circ}$ & $100.574^{\circ}$ & 1,750 & 193 & 2.46 & 0.25 & 103 \\
\hline 5 & $\begin{array}{l}\text { 石灰山 } \\
\text { Shihuishan }\end{array}$ & $\begin{array}{l}\text { 石灰山季雨林 } \\
\text { Limestone monsoon } \\
\text { forest }\end{array}$ & $21.911^{\circ}$ & $101.283^{\circ}$ & 606 & 258 & 3.36 & 0.90 & 56 \\
\hline 6 & $\begin{array}{l}\text { 新开地 } \\
\text { Xinkaidi }\end{array}$ & $\begin{array}{l}\text { 季节雨林次生林 } \\
\text { Secondary forest of } \\
\text { seasonal rainforest }\end{array}$ & $21.903^{\circ}$ & $101.275^{\circ}$ & 556 & 223 & 2.88 & 0.78 & 75 \\
\hline 7 & $\begin{array}{l}\text { 茶厂后山 } \\
\text { Chachanghoushan }\end{array}$ & $\begin{array}{l}\text { 季节雨林 } \\
\text { Seasonal rainforest }\end{array}$ & $22.155^{\circ}$ & $100.675^{\circ}$ & 784 & 169 & 2.21 & 0.49 & 129 \\
\hline 8 & $\begin{array}{l}\text { 44公里 } \\
44 \text { Gongli }\end{array}$ & $\begin{array}{l}\text { 季节雨林 } \\
\text { Seasonal rainforest }\end{array}$ & $21.971^{\circ}$ & $101.148^{\circ}$ & 806 & 287 & 3.58 & 1.07 & 49 \\
\hline 9 & $\begin{array}{l}\text { 预仑水库 } \\
\text { Menglunshuiku }\end{array}$ & $\begin{array}{l}\text { 季节雨林 } \\
\text { Seasonal rainforest }\end{array}$ & $21.935^{\circ}$ & $101.179^{\circ}$ & 656 & 361 & 4.56 & 0.72 & 30 \\
\hline 10 & $\begin{array}{l}\text { 江边站 } \\
\text { Jiangbianzhan }\end{array}$ & $\begin{array}{l}\text { 季节雨林 } \\
\text { Seasonal rainforest }\end{array}$ & $22.219^{\circ}$ & $100.734^{\circ}$ & 640 & 407 & 5.24 & 1.03 & 23 \\
\hline
\end{tabular}
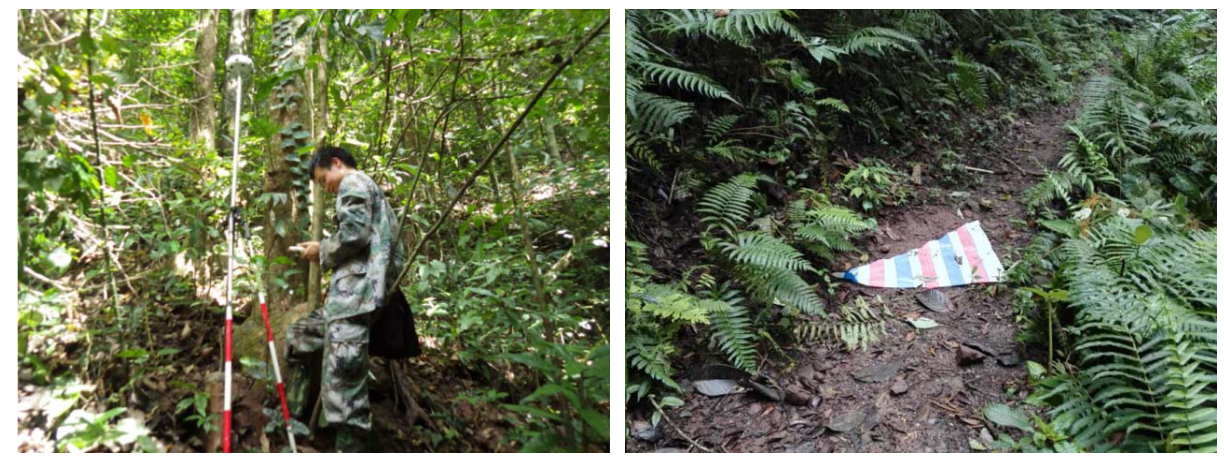

图2 森林中地面控制点坐标测量(左)与标识布设(右)示例

Fig. 2 Ground control point coordinate measurement (left) and mark (right) in forest
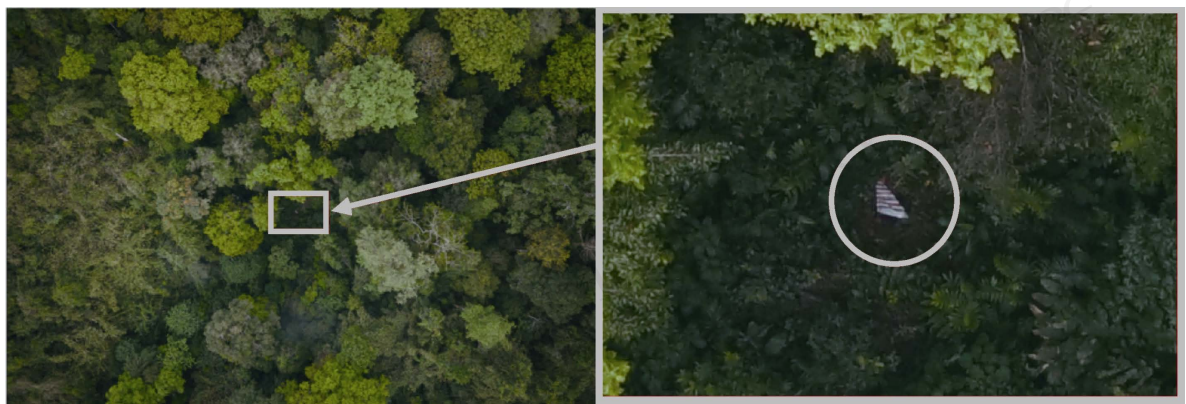

图3 航拍影像中的地面控制点标识示例

Fig. 3 Mark of ground control point in aerial photograph 
$30 \mathrm{~mm}$ 焦距下进行拍摄; 机型二为DJI M600 Pro (大 疆创新科技有限公司, 深圳, 中国)配A3 Pro飞控 (大疆创新科技有限公司, 深圳, 中国), 挂载A7R相 机(索尼株式会社, 东京, 日本)和35 mm定焦镜头 进行拍摄。机型一和机型二分别使用ZERO-GCS和 DJI GS Pro地面站进行航线规划和控制, 航向上照 片重叠率一般大于 $80 \%$, 航线间照片重叠率一般大 于 $60 \%$ 。

由于具体配置上的原因, 两个机型均未能直接 由飞控导出曝光点的位置数据, 因此我们通过外挂 的HOLUX M-241(长天科技股份有限公司, 新竹, 中国台湾)GPS记录仪对照片曝光点坐标进行记录。 使用时先将相机与GPS进行准确对时, 之后将GPS 调至1次/s的频率记录坐标。飞行任务完成后，通过 Holux Logger Utility (v1.1.0.48)软件将对应时刻的 照片与位置信息叠加并处理。

\section{3 无人机影像处理与点云生成}

本研究使用Photoscan软件(Agisoft LCC, 圣彼 得堡, 俄罗斯)对所得照片进行点云解算。该软件是 目前应用较多的一款摄影测量建模软件, 其解算精 度已得到业内的广泛检验(Turner et al, 2014; Gross \& Heumann, 2016; Jaud et al, 2016), 并已在考古 (Katz \& Friess, 2014)、测绘(Ryan et al, 2015)、农业 (Jin et al, 2017)、林业(Jensen \& Mathews, 2016)等多 个学科中得到应用。早期的摄影测量软件中控制点 精度并不参与建模过程, 但在v1.2.6及之后版本的
Photoscan中，控制点的实测精度同时也作为建模时 的基本输入参数之一参与模型解算，而软件在解算 完毕后也会结合照片重叠度等其他要素的综合信 息，重新给出每个点在模型中的估算精度(Agisoft, 2018), 这也为缺乏单独检查点时的模型精度检查 提供了基本参考。

软件使用时，手工输入每个地面控制点的经纬 度、高程和精度，在对应照片上手工标出对应控制 点位置, 之后由软件自动建立整个区域的点云和三 角网模型，并对控制点的实测精度和估算精度进行 比较。照片曝光点的重投影精度由软件自动解算生 成。本研究使用Photoscan v1.2.6在禁用成对预选 (pair preselection: disabled)的高精度模式(accuracy: high)对照片进行对齐处理(align photos)并生成中等 质量(quality: medium)的密集点云(dense cloud), 点 云密度见表1。Photoscan软件数据基本处理流程参 见图4。

\section{4 数字地形模型生产}

本研究使用两种方式对地形进行建模：一是估 测数字地形模型(estimated digital terrain model,

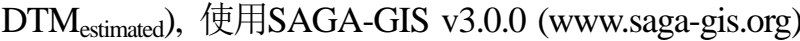
将点云转换为 $1 \mathrm{~m}$ 分辨率的栅格数据并建立数字表 面模型(digital surface model, DSM), 以DTM filer (slope-based)工具对点云中的地形点进行提取, 并 用多级B-样条插值法(multilevel B-spline interpolation)对所提地形点进行插值并建立栅格。考虑到样

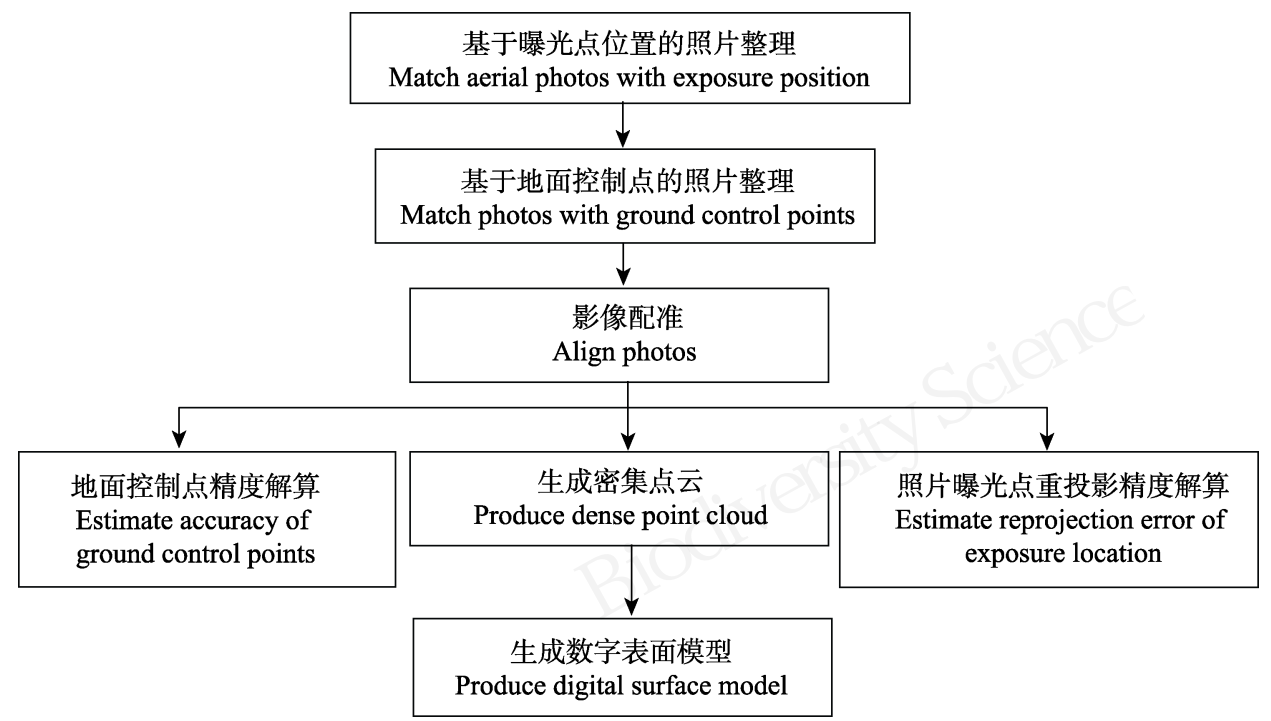

图4 基于Photoscan软件的航片数据处理流程

Fig. 4 Aerial photograph processing flow with Photoscan software 
地建设时常以 $20 \mathrm{~m} \times 20 \mathrm{~m}$ 为基本调查单元，以 $10 \mathrm{~m}$ $\times 10 \mathrm{~m}$ 作为全站仪测量的最小单元, 且全部样地均 属山区的坡地地形, 因此在DTM filer中设置查找半 径为 $10 \mathrm{~m}$, 大致坡度(approximate terrain slope)为 $30^{\circ}$ 。

二是实测数字地形模型(measured digital terrain

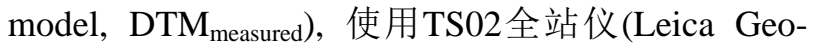
systems AG, Heerbrugg, Switzerland)按照 $10 \mathrm{~m} \times 10$ $\mathrm{m}$ 的网格对样地内地形进行实地测量, 之后将实测 点坐标导入ArcGIS v10.2 (Esri, Redlands, 美国), 以 克里格插值法(Kriging interpolation)对样地范围内 的地形进行插值并建立栅格。两种建模结果示意参 见图5。

\section{5 模型精度验证}

使用ArcGIS v10.2对实测地形模型和估测数字 地形模型中对应数据进行提取。数据提取时, 将样地 划分为 $1 \mathrm{~m} \times 1 \mathrm{~m}$ 的网格, 提取对应坐标点上的估测 数字地形模型和实测数字地形模型数值, 求取差值
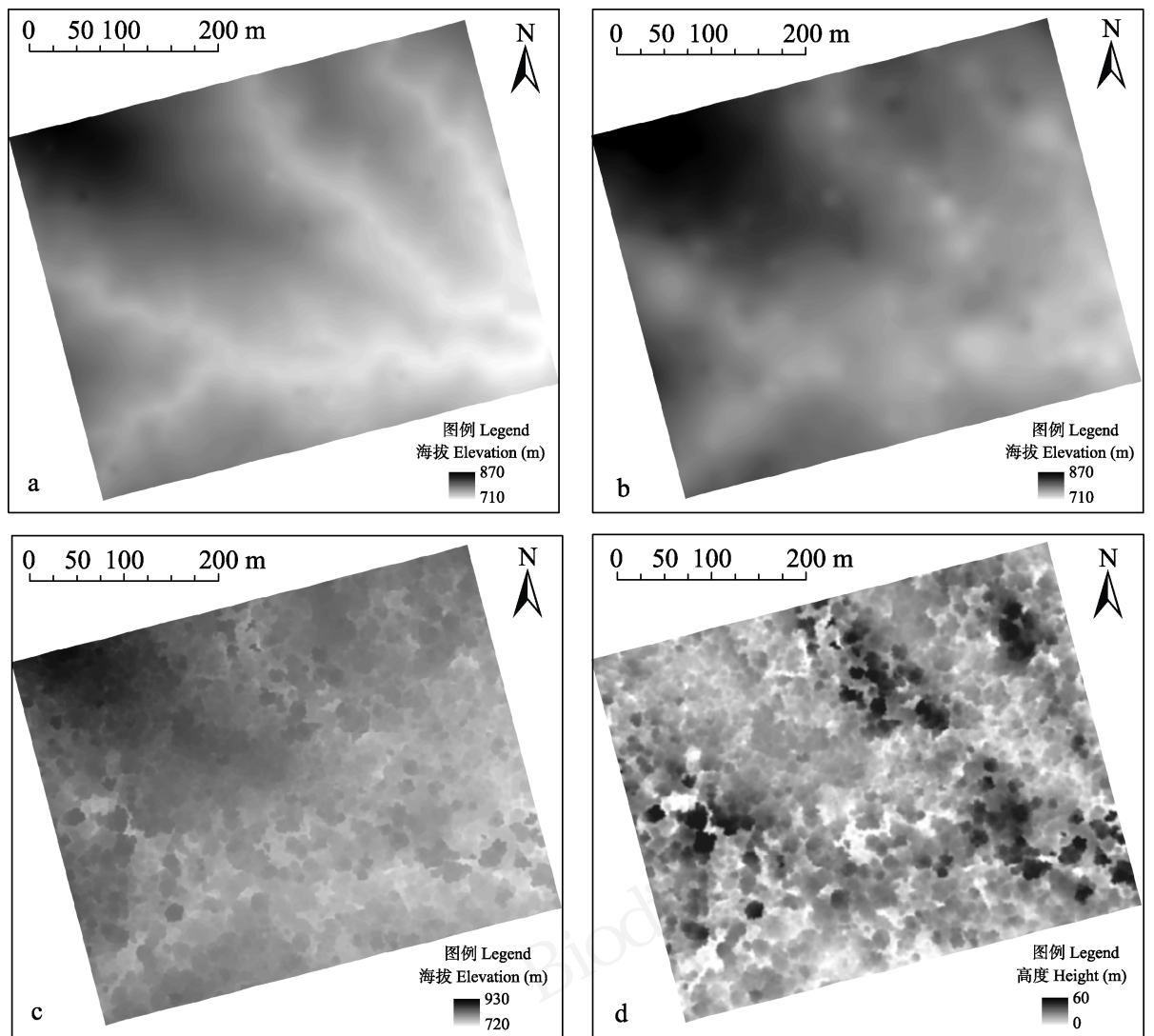

得到该点上的模型高差, 使用Excel 2016 (Microsoft Corp., Redmond, 美国)计算样地范围内所有模型高 差数据的平均值和标准差。

通过线性回归方程对实测地形模型和估测数 字地形模型间的拟合关系进行判断并以此验证模 型精度:

$$
y=k x+b
$$

式中, $y$ 为估测数字地形模型上提取的数值, $x$ 为实测 数字地形模型上提取的数值, $k$ 为方程斜率, $b$ 为常 数项。估测模型与实测模型相似程度越高, 则方程 斜率 $k$ 越趋近于 1 。

\section{6 森林参数提取}

使用ArcGIS v10.2对实测数字地形模型和数字 表面模型中对应数据进行提取。取 $1 \mathrm{~m} \times 1 \mathrm{~m}$ 栅格 4 个顶角处数字表面模型与实测数字地形模型间高 差的平均值作为该 $1 \mathrm{~m} \times 1 \mathrm{~m}$ 网格的模型高差。本研 究中首先去除模型高差序列由高至低排序中的后 $0.5 \%$ 极值, 并以此时高差值作为地面零点归零并推

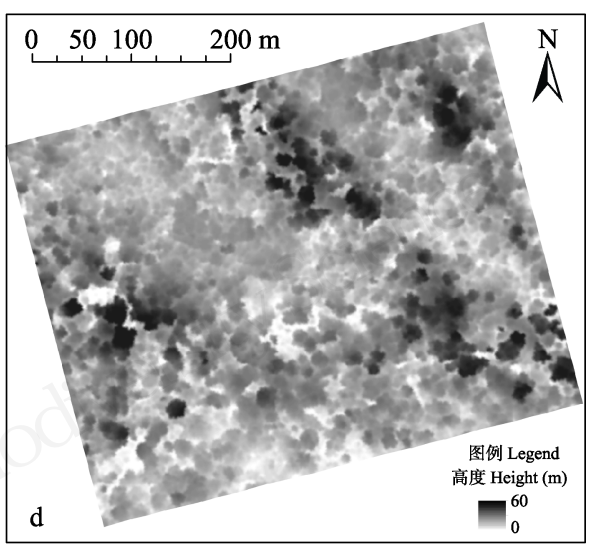

图5 实测数字地形模型(a)、估测数字地形模型(b)、数字表面模型(c)和林冠高度模型(d)示意图

Fig. 5 Example of measured digital terrain model (a), estimated digital terrain model (b), digital surface model (c) and canopy height model (d) 
算其他位置的林冠高度; 同时去除前 $0.5 \%$ 极值以消 除个别异常点对数据整体的影响。本研究依据Hubbell等(1999)对林窗的定义: 认为5 $\mathrm{m}$ 的林冠高度是 林窗和非林窗环境的分界, 并以此计算 $5 \mathrm{~m}$ 以上林 冠在整个样地中所占的面积比例, 即林冠覆盖度。

\section{7 数据分析}

本研究以成对样本 $t$ 检验对各因子间的差异程 度进行检验, 使用Pearson相关系数 (r) 对数据间的 相关性进行描述。使用逐步回归法在控制点数量、

飞行高度和RTK均方根误差等因子中篮选出点云 解算所得模型 $R M S E$ 的拟合度最优方程作为最优模 型进行讨论, 以赤池信息量准则 (Akaike information criterion, AIC)最低者为最优模型。模型所用的 自变量和因变量均经过标准化处理并以方差膨胀 因子(variance inflation factor, VIF)对自变量的共线 性程度进行检验。本研究中的成对样本 $t$ 检验和相关 性分析使用IBM SPSS Statistics v22.0 (IBM Corp., Armonk，美国)完成，逐步回归使用 R v3.4.0 (www.r-project.org/) 完成, 作图和 RMSE 计算使用 Excel 2016完成。

\section{3 结果}

\section{1 地面控制点测量精度}

本研究所选样地均为森林样地, 虽然控制点多
设置于林内相对开阔的林窗处，但由于当地森林树 木高大 (一般大于 $20 \mathrm{~m})$, 而林窗大小毕竟有限, RTK 系统在定位时不一定能够找到足够数量卫星，因此 导致定位精度较林外开阔地差，水平方向和垂直方 向上的 $R M S E$ 分别为 $0.167 \pm 0.158 \mathrm{~m}$ 和 $0.297 \pm 0.170 \mathrm{~m}$, 垂直误差大于水平误差(成对样本 $t$ 检验, $t=9.972, P$ $<0.01$ ) (表2)。

为便于后期数据融合, 测量地面控制点的同时 也使用RTK系统对样地的 4 个顶点进行坐标复核。 顶点桩处的水平方向 $R M S E$ 为 $0.392 \pm 0.368 \mathrm{~m}$, 垂直 方向 $R M S E$ 为 $0.657 \pm 0.412 \mathrm{~m}$, 仍然表现为垂直误差 大于水平误差(成对样本 $t$ 检验, $t=9.974, P<0.01$ )。 若单独对林窗处的地面控制点和样地顶点的误差 进行对比，则发现二者的水平方向 RMSE差异并不 显著(成对样本 $t$ 检验, $t=-1.778, P=0.109$ ), 但林下 样地顶点在垂直方向上的 RMSE显著大于林窗处的 地面控制点(成对样本 $t$ 检验, $t=-2.578, P=0.030$ ), 这可能是由于样地建设时多倾向于选择干扰较少 的森林地段, 因此样地顶点位置也很难遇到开阔林 窗，此时RTK系统的定位精度较林窗中更差。

\section{2 点云解算精度}

各样地的点云解算所得模型的垂直方向 RMSE 为 $0.62 \pm 0.62 \mathrm{~m}$, 水平方向 $R M S E$ 为 $0.54 \pm 0.41 \mathrm{~m}$, 垂直误差大于水平误差, 但差异并不显著(成对样

表2 样地控制点与样地顶点均方根误差

Table 2 RMSE of ground control point and corner stake in sample plots

\begin{tabular}{|c|c|c|c|c|c|c|c|c|}
\hline \multirow{2}{*}{$\begin{array}{l}\text { 样地名称 } \\
\text { Plot name }\end{array}$} & \multicolumn{4}{|c|}{ 地面控制点RMSE RMSE of ground control point } & \multicolumn{4}{|c|}{ 样地顶点RMSE RMSE of corner stake } \\
\hline & $\begin{array}{l}\text { 数量 } \\
\text { Number }\end{array}$ & $\begin{array}{l}\text { 垂直 } \\
\text { Vertical (m) }\end{array}$ & $\begin{array}{l}\text { 水平 } \\
\text { Horizontal (m) }\end{array}$ & $\begin{array}{l}\text { 全局 } \\
\text { Total (m) }\end{array}$ & $\begin{array}{l}\text { 数量 } \\
\text { Number }\end{array}$ & $\begin{array}{l}\text { 垂直 } \\
\text { Vertical (m) }\end{array}$ & $\begin{array}{l}\text { 水平 } \\
\text { Horizontal (m) }\end{array}$ & $\begin{array}{l}\text { 全局 } \\
\text { Total (m) }\end{array}$ \\
\hline 补蚌 Bubeng & 10 & 0.581 & 0.454 & 0.476 & 4 & 0.891 & 0.659 & 0.846 \\
\hline 过门山 Guomenshan & 6 & 0.247 & 0.057 & 0.076 & 4 & 0.833 & 0.634 & 0.648 \\
\hline 茶地头 Chaditou & 8 & 0.404 & 0.324 & 0.368 & 4 & 0.125 & 0.007 & 0.012 \\
\hline 大平掌 Dapingzhang & 10 & 0.333 & 0.202 & 0.260 & 4 & 0.633 & 0.336 & 0.532 \\
\hline 石灰山 Shihuishan & 7 & 0.086 & 0.005 & 0.007 & 4 & 0.671 & 0.311 & 0.560 \\
\hline 新开地 Xinkaidi & 7 & 0.205 & 0.090 & 0.091 & 4 & 0.284 & 0.022 & 0.100 \\
\hline $\begin{array}{l}\text { 茶厂后山 } \\
\text { Chachanghoushan }\end{array}$ & 7 & 0.104 & 0.006 & 0.009 & 4 & 0.293 & 0.020 & 0.104 \\
\hline 44公里 44 Gongli & 5 & 0.131 & 0.007 & 0.013 & 4 & 1.430 & 1.101 & 1.700 \\
\hline $\begin{array}{l}\text { 㐨仑水库 } \\
\text { Menglunshuiku }\end{array}$ & 5 & 0.487 & 0.292 & 0.406 & 4 & 1.082 & 0.691 & 0.994 \\
\hline 江边站 Jiangbianzhan & 7 & 0.397 & 0.228 & 0.310 & 4 & 0.325 & 0.139 & 0.161 \\
\hline 最大 Maximum & 10 & 0.581 & 0.454 & 0.476 & 4 & 1.430 & 1.101 & 1.700 \\
\hline 最小 Minimum & 5 & 0.086 & 0.005 & 0.007 & 4 & 0.125 & 0.007 & 0.012 \\
\hline $\begin{array}{l}\text { 平均值 } \pm \text { 标准差 } \\
\text { Mean } \pm \mathrm{SD}\end{array}$ & $7 \pm 2$ & $0.297 \pm 0.170$ & $0.167 \pm 0.158$ & $0.202 \pm 0.182$ & $4 \pm 0$ & $0.657 \pm 0.412$ & $0.392 \pm 0.368$ & $0.566 \pm 0.523$ \\
\hline
\end{tabular}


本 $t$ 检验, $t=0.374, P=0.717)$ 。通过逐步回归进一步 考虑控制点数量、飞行高度和地面控制点精度对点 云解算模型中均方根误差的影响后发现，所有模型 中自变量的VIF均小于 4 , 模型不存在多重共线性问 题，点云的全局解算精度主要受控制点地面测量精 度和控制点数量的影响, 垂直方向上的规律亦与此 类似(表3)。水平方向 $R M S E$ 回归模型 $P=0.093$, 大于 常用的 0.05 的显著水平, 因此虽此时有模型参数, 但该模型并不具有特别强的统计学意义; 即使对于 此时模型中的 $x_{1}$ (控制点数量)而言, 虽其为正值, 但对该项的显著性检验结果也并不明显(表3), 因此 此时 $x_{1}$ 的斜率并不具有明确的统计学意义, 并不能 因此认为水平方向的 $R M S E$ 与控制点数量呈正相关 关系，而该问题的原因可能与本文中各样地间的控 制点数量变化不大有关。

\section{3 照片曝光点重投影误差}

本研究主要使用地面控制点对影像进行配准, 因此相机曝光点坐标对最终点云数据解算的贡献 实际上极为有限。但为对无地面控制点时的测量误 差进行探讨, 在此仍将曝光点的误差情况进行分析 以供参考。

相对地面控制点的测量误差，曝光点的重投影 误差始终处于一个比较高的水平(表4), 水平方向 $R M S E$ 为 $18.434 \pm 5.252 \mathrm{~m}$, 垂直方向 $R M S E$ 为34.042 $\pm 6.920 \mathrm{~m}$ ，这相对于地面RTK的定位精度而言已经 属于数量级上的差异。曝光点重投影的垂直误差显
表3 控制点数量 $\left(x_{1}\right)$ 、飞行高度 $\left(x_{2}\right)$ 和对应方向上的RTK均方 根误差 $\left(x_{3}\right)$ 对点云解算模型中均方根误差的逐步回归结果

Table 3 Stepwise regression analysis result of ground control point amount $\left(x_{1}\right)$, flying height $\left(x_{2}\right)$, and root mean square error of RTK in related direction $\left(x_{3}\right)$ to root mean square error (RMSE) in estimated model (point cloud based)

\begin{tabular}{|c|c|c|c|}
\hline$y$ & $\begin{array}{l}\text { 模型垂直均方根 } \\
\text { 误差 } \\
\text { Vertical RMSE in } \\
\text { estimated model }\end{array}$ & $\begin{array}{l}\text { 模型水平均方根 } \\
\text { 误差 } \\
\text { Horizontal RMSE } \\
\text { in estimated model }\end{array}$ & $\begin{array}{l}\text { 模型整体均方根 } \\
\text { 误差 } \\
\text { Total RMSE in } \\
\text { estimated model }\end{array}$ \\
\hline $\begin{array}{l}\text { 截距 } \\
\text { Intercept }\end{array}$ & $-3.336 \mathrm{E}-16$ & $4.430 \mathrm{E}-17$ & $-1.540 \mathrm{E}-16$ \\
\hline$x_{1}$ & $-0.309 *$ & 0.082 & -0.176 \\
\hline$x_{2}$ & -0.005 & $0.003^{*}$ & \\
\hline$x_{3}$ & $4.964 *$ & & $3.157^{* *}$ \\
\hline$V I F \_x_{1}$ & 2.022 & 1.026 & 1.237 \\
\hline$V I F \_x_{2}$ & 3.285 & 1.026 & \\
\hline$V I F \_x_{3}$ & 3.775 & & 1.237 \\
\hline$R^{2}$ & 0.757 & 0.493 & 0.650 \\
\hline $\begin{array}{l}F \text {-统计量 } \\
F \text {-statistic }\end{array}$ & 6.245 & 3.405 & 6.504 \\
\hline$P$ & 0.028 & 0.093 & 0.025 \\
\hline
\end{tabular}

著高于水平误差(成对样本 $t$ 检验, $t=9.974, P<$ $0.01)$ ，相关分析表明，曝光点水平方向 $R M S E$ 与飞 行高度间无明显相关关系 $(r=0.052, P>0.05)$, 但 曝光点的垂直方向 $R M S E$ 与飞行高度呈显著相关 $(r$ $=-0.729, P<0.05)$ 。这可能与单点GPS大多侧重于 确保平面坐标精度，而测高精度相对较低，测得稳 定高程的耗时较长有关。

表4 各样地相机曝光点重投影误差

Table 4 Reprojection error of camera exposure position in sample plots

\begin{tabular}{lllll} 
样地名称 Plot name & 照片数量 Number of photos & \multicolumn{2}{c}{$R M S E$} & 全局 Total (m) \\
\cline { 3 - 5 } & & 垂直 Vertical (m) & 水平 Horizontal (m) & 34.733 \\
\hline 补蚌 Bubeng & 1,142 & 28.924 & 19.230 & 25.356 \\
过门山 Guomenshan & 1,632 & 21.951 & 12.690 & 39.336 \\
茶地头 Chaditou & 625 & 32.228 & 22.554 & 39.101 \\
大平掌 Dapingzhang & 858 & 36.709 & 13.466 & 45.724 \\
石灰山 Shihuishan & 1,536 & 39.100 & 23.704 & 45.510 \\
新开地 Xinkaidi & 1,293 & 37.688 & 25.509 & 50.839 \\
茶厂后山 Chachanghoushan & 2,088 & 47.508 & 18.102 & 36.922 \\
44公里 44 Gongli & 2,013 & 34.682 & 12.663 & 35.028 \\
秒仑水库 Menglunshuiku & 675 & 32.660 & 12.659 & 37.469 \\
江边站 Jiangbianzhan & 1,132 & 28.973 & 23.759 & 50.839 \\
最大 Maximum & 2,088 & 47.508 & 25.509 & 25.356 \\
最小 Minimum & 625 & 21.951 & 12.659 & $39.002 \pm 7.108$ \\
平均值 \pm 标准差 Mean \pm SD & $1,299 \pm 515$ & $34.042 \pm 6.920$ & $18.434 \pm 5.252$ & \\
\hline
\end{tabular}


表5 线性方程检验结果与样地结构特征

Table 5 Linear equation test result and structure characteristics of sample plots

\begin{tabular}{|c|c|c|c|c|c|c|c|c|c|}
\hline \multirow[t]{2}{*}{$\begin{array}{l}\text { 样地名称 } \\
\text { Plot name }\end{array}$} & \multirow[t]{2}{*}{$\begin{array}{l}\text { 面积 } \\
\text { Area } \\
\text { (ha) }\end{array}$} & \multirow[t]{2}{*}{$\begin{array}{l}\text { 林冠覆盖度 } \\
\text { Canopy cover }\end{array}$} & \multirow[t]{2}{*}{$\begin{array}{l}\text { 林冠最大高度 } \\
\text { rMaximum } \\
\text { height of } \\
\text { canopy (m) }\end{array}$} & \multirow[t]{2}{*}{$\begin{array}{l}\text { 林冠平均高度 } \\
\text { Average height } \\
\text { of canopy (m) }\end{array}$} & \multirow[t]{2}{*}{$\begin{array}{l}\text { 估测地形与实测地 } \\
\text { 形高差均值 } \\
\text { Mean difference } \\
\text { between DTM } \\
\text { and DTM } \\
\text { measured }\end{array}$} & \multirow{2}{*}{$\begin{array}{l}\text { 估测地形与实测地 } \\
\text { 形高差标准差 } \\
\text { Standard deviation } \\
\text { between DTM } \text { estimated }_{\text {and DTM }} \\
\text { ameasured }\end{array}$} & \multicolumn{3}{|c|}{$\begin{array}{l}\text { 估测地形模型 }(y) \text { 与实测地形模型 } \\
(x) \text { 的检验方程参数对比 } \\
\text { Linear equation test result of } \\
\operatorname{DTM}_{\text {estimated }}(y) \text { and DTM measured }(x)\end{array}$} \\
\hline & & & & & & & $k$ & $b$ & $R^{2}$ \\
\hline $\begin{array}{l}\text { 补蚌 } \\
\text { Bubeng }\end{array}$ & 20 & 0.90 & 54.67 & 22.43 & 10.06 & 6.09 & 0.995 & 14.187 & 0.981 \\
\hline $\begin{array}{l}\text { 过门山 } \\
\text { Guomenshan }\end{array}$ & 20 & 0.97 & 52.10 & 22.90 & 2.10 & 6.70 & 1.005 & -2.782 & 0.992 \\
\hline $\begin{array}{l}\text { 茶地头 } \\
\text { Chaditou }\end{array}$ & 1 & 0.95 & 35.63 & 16.79 & 4.55 & 5.90 & 0.979 & 30.783 & 0.761 \\
\hline $\begin{array}{l}\text { 大平掌 } \\
\text { Dapingzhang }\end{array}$ & 1 & 0.93 & 37.66 & 18.37 & -2.91 & 5.28 & 0.888 & 188.821 & 0.920 \\
\hline $\begin{array}{l}\text { 石灰山 } \\
\text { Shihuishan }\end{array}$ & 1 & 0.75 & 22.82 & 8.22 & 0.50 & 2.27 & 0.982 & 11.895 & 0.978 \\
\hline $\begin{array}{l}\text { 新开地 } \\
\text { Xinkaidi }\end{array}$ & 1 & 0.90 & 20.37 & 11.68 & 2.79 & 3.94 & 1.199 & -108.789 & 0.788 \\
\hline $\begin{array}{l}\text { 茶厂后山 } \\
\text { Chachang- } \\
\text { houshan }\end{array}$ & 1 & 0.98 & 48.98 & 24.44 & 7.62 & 6.37 & 0.839 & 125.731 & 0.834 \\
\hline $\begin{array}{l}\text { 44公里 } \\
44 \text { Gongli }\end{array}$ & 1 & 0.99 & 44.80 & 25.36 & -3.60 & 8.58 & 1.237 & -196.197 & 0.761 \\
\hline $\begin{array}{l}\text { 预仑水库 } \\
\text { Menglun- } \\
\text { shuiku }\end{array}$ & 1 & 0.89 & 45.79 & 23.82 & -26.48 & 7.42 & 0.973 & -7.650 & 0.779 \\
\hline $\begin{array}{l}\text { 江边站 } \\
\text { Jiangbianzhan }\end{array}$ & 1 & 0.93 & 52.46 & 26.43 & 0.18 & 17.12 & 1.245 & -159.057 & 0.638 \\
\hline
\end{tabular}

\section{4 地形建模精度}

对比基于无人机摄影测量建立的估测数字地 形模型和基于样地实测高程建立的实测数字地形 模型后发现, 对于 20 ha级别的大样地, 估测地形与 实测地形间拟合关系相对较好, 补蛙和过门山的检 验方程斜率 $k$ 分别为 0.995 和 1.005 , 接近理想值 1 ; 此时两地的 $R^{2}$ 也分别达到 0.981 和 0.992 , 模型间的 拟合程度相对较好; 但是, 对于 1 ha尺度的样地, 除石灰山和大平掌样地 $R^{2}$ 分别达到 0.978 和 0.920 外, 其他样地的 $R^{2}$ 仅为 $0.638-0.834$, 拟合精度相对较低 (表5)。此外, 1 ha样地的检验方程斜率 $k$ 亦变化较大, 介于0.839-1.245之间。

除大平掌、44 公里和勐仑水库3块样地外, 多 数样地在估测地形模型中的平均海拔要高于实测 地形(表5)。这可能是由于摄影测量法难以直视地表, 易将空中看到的植被表面误判为地表, 因此导致模 型中的估测海拔高于实际值。少数样地中估测地形 高程小于实际地形的情况, 则有可能与用于校正的 地面控制点数量不足和分布不均有关。
相关分析结果表明，估测地形与实测地形间高 差的标准差与林冠平均高度成显著的正相关关系 $(r=0.713, P<0.05)$, 林冠平均高度越高, 则估测地 形误差的变化范围越大。由于林冠平均高度同时也 与林冠覆盖度相关 $(r=0.721, P<0.05)$, 因此在利 用摄影测量法对地形建模的过程中, 应尽量避免在 植被高大且茂密的区域做直接应用。

\section{4 讨论}

\section{1 地面测量坐标误差来源与分析}

影像地面分辨率、镜头畸变和控制点精度是摄 影测量的主要误差来源(冯茂平等, 2017)。其中, 地 面分辨率主要与成像质量有关，可通过控制飞行高 度和提升相机像素等方法解决; 镜头畸变会造成像 点位移，可通过提高照片重叠度，软件非量测校正 模型等方法处理(Brown，1971; 黄应清等，2015); 地面控制点的测量精度由于受许多难以控制的外 界因素影响，因此成为外业操作中可能对后期解算 造成误差的最主要因素。 
全球导航卫星系统是目前主流的地面导航定 位方式。但是, 不论是GPS还是其他GNSS系统, 该 技术的应用基础都是需要接收机接收到足够多的 卫星的导航信号, 而当导航信号被干涉和阻隔而无 法收取或中断时, 其定位精度必然会受到影响(陈 俊勇和党亚民, 2005)。在高精度GNSS定位的各种误 差源中, 如星历误差、卫星钟差、大气折射误差和 接收机钟差等系统误差大多可以通过模型改正、参 数求取或差分技术等方式得到消除或减弱，但是， 由被测站附近的反射物反射卫星信号进入接收机 天线而导致的多路径效应误差由于在基线两端不 具有相关性(戴吾蛟等, 2008), 因此很难得到有效控 制, 多路径误差也因此成为卫星导航技术中主要的 误差来源(范晓燕和周乾, 2010)。

热带森林中林窗面积占森林面积的比例一般 为 3-25\% (Pickett \& White, 1985), 平均大小为 80-600 m² (Brokaw, 1985; Pickett \& White, 1985), 这些条件意味着即使选择在有一定可见天空的林 窗中布置地面控制点，也仍然面临着空间过于狭小 的问题。在本研究所涉及的样地中, 林冠最大高度 为20.37-54.67 m, 林冠覆盖度为0.75-0.99 (表5), 接收机周围大量的高䇯树木会不断反射卫星信号, 进而导致强烈的多路径效应, 干扰GNSS系统的正 常定位(Trajkovski et al, 2010; Ucar et al, 2014)。

本研究所选用的RTK技术是目前精确测量中 的常用技术之一, 其通过两个相同的GNSS接收机 同时在相邻地点进行观测求差，消除卫星导航信号 传递过程中大部分的相同误差项, 以此提高测量精 度(赵琳等, 2011)。该方法在正常工作时定位误差可 控制在厘米甚至毫米级别, 这相对传统的单点定位 误差而言已是有了极大的进步。但是，该技术在具 体应用时也需要满足一些前提条件, 即两个接收机 (移动站和基准站)需要共同观测一定数量的相同卫 星, 并通过数据链实时通讯以完成解算, 而解算误 差会随接收机间距离的增加而增加。在森林样地测 量的实际应用中, 由于林内很难找到开阔场地, 因 此RTK的基准站一般都只能设立于距样地一定距 离外的开阔场地以保证能够接收到足够多的卫星 信号与移动站进行匹配, 但这会导致两个接收机间 距离的增加, 有时还可能增加基准站和移动站间的 通讯难度(许多森林样地因建设在保护区内而缺乏 手机信号, 而选用电台通讯时又极易受地形遮挡和
距离限制)。同时，由于RTK本质仍然是卫星导航技 术的延伸，因此移动站在林内使用时亦会受到多路 径效应的强烈影响。

\section{2 控制点数量与误差}

控制点数量直接关系外业工作量大小, 因此也 是值得研究者根据自身条件仔细勘酌的问题之一。 目前测绘行业上对数字航空摄影测量及控制测量 已有严格规范(邓国庆等, 2011)，以飞行高度的 $1 / 5$ 到 $1 / 10$ 为间隔在测区均匀放置控制点被认为是控制 精度的理想方式(Harwin \& Lucieer, 2012)。但是，在 森林样地测量的实践中，由于林窗分布不均的关系， 找到开阔且不受森林生长影响的理想地面控制点 位置基本是可遇而不可求，很难按照规范均匀放置 足够多的地面控制点。过少的控制点可能会导致单 点误差在整体中的权重过大，一个不合格的定位点 就有可能导致整个数据结果出现较大偏差(Tomastik et al, 2017); 而较多的控制点数量虽然能够允 许模型的整体解算结果对单点误差变化不那么敏 感(James \& Robson, 2012), 但这又必须考虑布设时 额外的时间和人力投入。现有研究中, 研究者根据 各自的实践经验，使用的地面控制点数量从5-89个 不等，其精度多介于厘米到米之间(Tomastik et al, 2017)。有学者认为至少应有5个或以上的控制点作 为建模参考(Harwin et al, 2015), 也有研究认为8-9 个控制点是较易接受的范围(Tahar, 2013), 但总体 而言，对于森林近地面遥感过程中所需的地面控制 点数量, 一般都只能是在力所能及的范围内尽量满 足航摄规范要求。在本研究中, 控制点数量并未对 软件解算结果有显著影响, 这可能与本研究中各样 地间的控制点数量变化不大有关。

地面控制点的数量与其分布也有一定相关性。 一般来说，控制点应该立体、均匀地分布在测区内， 按照一定间距布设以覆盖所有的摄影基线，同时还 应注意加强测区边缘的控制并兼顾中部控制，点位 不能分布在近似一条直线上或者近似平面内(张剑 清等, 2003)。但是，当地面控制点数量较少时，必然 很难做到以上要求, 而这也很可能是导致预仑水库 测量案例中估测地形高程平均值大大低于实测地 形高程的主要原因(该样地实际测量时的 5 个控制点 中有 4 个集中分布于整个测区的西南侧，而样地实 际所在位置偏向测区东北部，附近仅有 1 个控制 点)。在条件允许时, 我们仍然建议在样地内及周围 
各个方向上尽可能地均匀布置足够数量的地面控 制点, 以获得相对理想的解算精度。

\section{3 曝光点坐标误差来源与分析}

虽然本研究在无人机上使用的是外挂GPS对曝 光位置进行记录, 但普通轻小型无人机上自带的 GNSS 定位精度一般也仅为2-10 m (Duffy et al, 2017), 若再考虑相机主光轴垂直程度、云台姿态误 差、曝光点距离地面高度等其他因素, 则类似本研 究的39.002 $\pm 7.108 \mathrm{~m}$ 的曝光点重投影误差也能够 理解。缺乏地面控制点坐标校正的轻小型无人机近 地面遥感数据可能很难直接与地面实测数据融合 使用。虽然本研究中的曝光点重投影位置并未实际 应用于解算误差校正，但在无人机平台应用的其他 一些案例中, 由于事发突然或是调查地点难以通达, 可能难以布设和测量准确的地面控制点坐标供后 期校正参考(Duffy et al, 2017), 则此时曝光点重投 影坐标的准确性就会显得非常重要。近年随着技术 的发展, GNSS的RTK和PPK (post processing kinematic，后差分)技术正与无人机平台结合并发展出 免像控测绘技术, 其通过在地面设立一个已知坐标 的基准站, 将无人机本身作为移动站, 配合机上惯 性测量单元(IMU)的姿态参数和精密的云台及相机 检校, 即可快速获取到相对准确的曝光点投影坐标 和地面像元坐标(范秀庆，2017; 田超等，2017)。目 前该技术主要在价格较高的工业级无人机上得到 应用, 未来可能会成为无人机近地面遥感中误差控 制技术的重要发展方向之一。

\section{4 地面高程模型精度分析}

摄影测量法虽然能够对表面高程进行建模, 但 由于其基本原理是基于已知点和角度的前端交汇 来求取未知点坐标, 因此每个需要解算位置的点上 都必须要能够在多张照片上被发现。已有研究发现, 虽然摄影测量法建立的点云精度近似于激光雷达, 但是由于相机始终无法透视林下, 前端交汇原理也 要求目标位置必须要被一定数量的照片重叠覆盖, 因此该方法在实际应用时更适合用于数字表面模 型的建立(Lisein et al, 2013)。由于摄影测量法所得 数据毕竟只是代表表面高程, 因此即使将一定范围 内的点云最低点作为地面点进行判断, 仍有可能由 于所见物为林下植被或是地面过暗无法直视而高 估地面高程(Duffy et al, 2017; Tomastik et al, 2017), 进而又导致对整体林冠高度的低估。同时, 摄影测
量法建模时容易忽略一些物体的细部特征(Lisein et al, 2013), 这也可能导致模型的误差增加。由于以上 条件制约, 现有的基于摄影测量的森林调查一般也 不直接使用摄影测量法估算的数字地形模型，而是 更倾向于将该方法建立的数字表面模型与地面实 测地形进行融合计算以获取实际的森林结构参数 (如: Zhang et al, 2016)。

本研究中, 基于摄影测量结果的20 ha样地估 测地形与实测地形间的拟合结果要优于多数的 1 ha 样地, 其原因可能就是大型样地相对小型样地能够 容纳更多的林窗，因此软件建模时选用的点云最低 点更接近于实际地面，而范围的扩大也使得有更多 的地面点供选择和参考。摄影测量法所直接估测的 地形结果在 20 ha 尺度下可能有一定的参考价值, 但我们在此并不建议在林冠平均高度和林冠覆盖 度均较高的森林地段上于小型样地中直接使用该 方法建立热带森林的地面高程模型。

\section{结论}

空间坐标匹配是机载观测数据与地面测量结 果间相互融合的关键步骤。森林样地由于其所处环 境的特殊性, 需要研究者在获取科学数据的过程中 考虑更多的误差控制问题。无论是无人机，还是 RTK/PPK，都是近年才得到大规模应用的新兴技术， 这些技术本身也还在不断地发展和完善过程中。现 有的GNSS定位技术在林下应用时仍然存在许多问 题，研究者需要根据自己的实际状况，选择合适的 方法, 在遥感数据中确定现有样地的坐标并对误差 进行控制。若不经地面控制点校正，单站式GNSS 的定位信息可能并不足以满足无人机平台数据与 地面数据融合的需要，因此使用时仍需注意在飞行 前事先布置足够数量和质量的地面控制点。摄影测 量法的优势在于能够以相对简单的前端设备获取 数据并建立数字表面模型, 但实际使用时应注意其 并不能够很好地对林下地形进行建模，尤其是在样 地面积较小或是林冠平均高度和林冠覆盖度较高 的情况之下。

致谢：本研究得到西双版纳国家级自然保护区管护 局和纳板河流域国家级自然保护区管理局的大力 支持，在此一并致谢。 


\section{参考文献}

Agisoft (2018) Agisoft PhotoScan User Manual. http://www. agisoft.com/downloads/user-manuals. (accessed on 2018-06-30)

Brokaw NVL (1985) Gap-phase regeneration in a tropical forest. Ecology, 66, 682-687.

Brown DC (1971) Close-range camera calibration. Photogrammetric Engineering, 37, 855-866.

Chen JY, Dang YM (2005) Recent progress in global navigation of satellite system. Science of Surveying and Mapping, 30(2), 9-12. (in Chinese with English abstract) [陈俊勇, 党 亚民 (2005) 全球导航卫星系统的新进展. 测绘科学, 30(2), 9-12.]

Christie KS, Gilbert SL, Brown CL, Hatfield M, Hanson L (2016) Unmanned aircraft systems in wildlife research: Current and future applications of a transformative technology. Frontiers in Ecology and the Environment, 14, 242-252.

Cunliffe AM, Brazier RE, Anderson K (2016) Ultra-fine grain landscape-scale quantification of dryland vegetation structure with drone-acquired structure-from-motion photogrammetry. Remote Sensing of Environment, 183, 129-143.

Dai WJ, Ding XL, Zhu JJ (2008) Study on multipath effect in structure health monitoring using GPS. Journal of Geodesy and Geodynamics, 28, 65-70. (in Chinese with English abstract) [戴吾蛟, 丁晓利, 朱建军 (2008) GPS动态变形测 量中的多路径效应特征研究. 大地测量与地球动力学, 28, 65-70.]

Deng GQ, Duan YH, Xiao XN, Wang ZH, Zhou Y, Jiang HB, Zhu J (2011) On the revision of AerophotogrammetryControl Survey Specifications. Standardization of Surveying and Mapping, 27(2), 3-9. (in Chinese with English abstract) [邓国庆, 段怡红, 肖学年, 王占宏, 周一, 蒋红兵, 朱健 (2011) 《数字航空摄影测量 控制测量规范》编写说明. 测绘标准化, 27(2), 3-9.]

Duffy JP, Cunliffe AM, DeBell L, Sandbrook C, Wich SA, Shutler JD, Myers-Smith IH, Varela MR, Anderson K (2017) Location, location, location: Considerations when using lightweight drones in challenging environments. Remote Sensing in Ecology and Conservation, 4, 7-19.

Fan XY, Zhou Q (2010) Review of multipath effects in GPS measurement. Chinese Journal of Engineering Geophysics, 7, 382-386. (in Chinese with English abstract) [范晓燕, 周 乾 (2010) 测量中多路径效应研究综述. 工程地球物理 学报, 7, 382-386.]

Fan XQ (2017) Application of UAV image free control technique in topographic map surveying. Bulletin of Surveying and Mapping, (Suppl.1), 66-68. (in Chinese with English abstract) [范秀庆 (2017) 无人机免像控技术在地形图测 量中的应用研究. 测绘通报, (增刊 1), 66-68.]

Feng MP, Zhao YP, Yang ZY, Zhang QG (2017) Research and application of key techniques for high precision mapping of 1:500 aerial photogrammetry. Surveying and Mapping, 40(4), 178-192. (in Chinese with English abstract) [冯茂平,
赵元沛, 杨正银, 张秦罡 (2017) 1:500 航测法高精度成 图的关键技术研究及其应用. 测绘, 40(4), 178-192.]

Gross JW, Heumann BW (2016) A statistical examination of image stitching software packages for use with unmanned aerial systems. Photogrammetric Engineering and Remote Sensing, 82, 419-425.

Harwin S, Lucieer A (2012) Assessing the accuracy of georeferenced point clouds produced via multi-view stereopsis from unmanned aerial vehicle (UAV) imagery. Remote Sensing, 4, 1573-1599.

Harwin S, Lucieer A, Osborn J (2015) The impact of the calibration method on the accuracy of point clouds derived using unmanned aerial vehicle multi-view stereopsis. Remote Sensing, 7, 11933-11953.

Huang YQ, Wen J, Xie ZH (2015) Research on non-metric calibration algorithm of camera lens distortion. Modern Electronics Technique, 38(20), 59-66. (in Chinese with English abstract) [黄应清, 文军, 谢志宏 (2015) 摄像机 畸变的非量测校正方法研究. 现代电子技术, 38(20), 59-66.]

Hubbell SP, Foster RB, O’Brien ST, Harms KE, Condit R, Wechsler B, Wright SJ, de Lao SL (1999) Light-gap disturbances, recruitment limitation, and tree diversity in a neotropical forest. Science, 283, 554-557.

Husson E, Ecke F, Reese H (2016) Comparison of manual mapping and automated object-based image analysis of non-submerged aquatic vegetation from very-high- resolution UAS Images. Remote Sensing, 8, 724.

James MR, Robson S (2012) Straightforward reconstruction of 3D surfaces and topography with a camera: Accuracy and geoscience application. Journal of Geophysical Research, 117, F03017.

Jaud M, Passot S, Le Bivic R, Delacourt C, Grandjean P, Le Dantec N (2016) Assessing the accuracy of high resolution digital surface models computed by PhotoScan ${ }^{\circledR}$ and MicMac ${ }^{\circledR}$ in sub-optimal survey conditions. Remote Sensing, 8, 465.

Jensen JLR, Mathews AJ (2016) Assessment of image-based point cloud products to generate a bare earth surface and estimate canopy heights in a woodland ecosystem. Remote Sensing, 8, 50.

Jin XL, Liu SY, Baret F, Hemerle M, Comar A (2017) Estimates of plant density of wheat crops at emergence from very low altitude UAV imagery. Remote Sensing of Environment, 198, 105-114.

Katz D, Friess M (2014) Technical note: 3D from standard digital photography of human crania-A preliminary assessment. American Journal of Physical Anthropology, 154, 152-158.

Koh LP, Wich SA (2012) Dawn of drone ecology: Low-cost autonomous aerial vehicles for conservation. Tropical Conservation Science, 5, 121-132.

Li Y, Xu W, Wu W (2011) Application research on aviation 
remote sensing UAV for disaster monitoring. Journal of Catastrophology, 26(1), 138-143. (in Chinese with English abstract) [李云, 徐伟, 吴玮 (2011) 灾害监测无人机技术 应用与研究. 灾害学, 26(1), 138-143.]

Lisein J, Pierrot-Deseilligny M, Bonnet S, Lejeune P (2013) A photogrammetric workflow for the creation of a forest canopy height model from small unmanned aerial system imagery. Forests, 4, 922-944.

Ma RS, Ma SQ, Wang LP, Zhang XP, Pan Y, Guan FS (2008) Preliminary experiment of forest fire monitoring system on unmanned aerial vehicle. Meteorological Science and Technology, 36, 100-104. (in Chinese with English abstract) [马瑞升, 马舒庆, 王利平, 张小平, 潘毅, 官福顺 (2008) 微型无人驾驶飞机火情监测系统及其初步试验. 气象科 技, 36, 100-104.]

Pickett STA, White PS (1985) The Ecology of Natural Disturbance and Patch Dynamics. Academic Press, New York.

Ryan JC, Hubbard AL, Box JE, Todd J, Christoffersen P, Carr JR, Holt TO, Snooke N (2015) UAV photogrammetry and structure from motion to assess calving dynamics at Store Glacier, a large outlet draining the Greenland ice sheet. Cryosphere, 9, 1-11.

Tahar KN (2013) An evaluation of different number of ground control points in unmanned aerial vehicle photogrammetric block. In: International Archives of the Photogrammetry, Remote Sensing and Spatial Information Sciences (eds ISPRS 8th 3DGeoInfo Conference \& WG II/2 Workshop), pp. 93-98. ISPRS, Istanbul.

Tian C, Chen J, Li NN, Guo YH (2017) An rapid method to build high accuracy DSM with UAV image free control technique. Bulletin of Surveying and Mapping, (8), 158160. (in Chinese with English abstract) [田超, 陈杰, 李能 能, 郭耀煌 (2017) 利用无人机免像控快速构建高精度 DSM. 测绘通报, (8), 158-160.]

Tomastik J, Mokros M, Salon S, Chudy F, Tunak D (2017) Accuracy of photogrammetric UAV-based point clouds under conditions of partially-open forest canopy. Forests, 8, 151.

Trajkovski KK, Sterle O, Stopar B (2010) Sturdy positioning with high sensitivity GPS sensors under adverse conditions. Sensors, 10, 8332-8347.

Turner D, Lucieer A, Wallace L (2014) Direct georeferencing of ultrahigh-resolution UAV Imagery. IEEE Transactions on Geoscience and Remote Sensing, 52, 2738-2745.

Ucar Z, Bettinger P, Weaver S, Merry KL, Faw K (2014) Dynamic accuracy of recreation-grade GPS receivers in oak-hickory forests. Forestry, 87, 504-511.

Westoby MJ, Brasington J, Glasser NF, Hambrey MJ, Reynolds JM (2012) 'Structure-from-Motion’ photogrammetry: A low-cost, effective tool for geoscience applications. Geomorphology, 179, 300-314.

Zhang J, Hu JB, Lian JY, Fan ZJ, Ouyang XJ, Ye WH (2016) Seeing the forest from drones: Testing the potential of lightweight drones as a tool for long-term forest monitoring. Biological Conservation, 198, 60-69.

Zhang JQ, Pan L, Wang SG (2003) Phototopography. Wuhan University Press, Wuhan. (in Chinese) [张剑清, 潘励, 王 树根 (2003) 摄影测量学. 武汉大学出版社, 武汉.]

Zhang Y, Tao P, Liang SX, Liang WL (2011) Research on application of UAV RS techniques in forest inventories. Journal of Southwest Forestry University, 31(3), 49-53. (in Chinese with English abstract) [张园, 陶萍, 梁世祥, 梁万 里 (2011) 无人机遥感在森林资源调查中的应用. 西南 林业大学学报, 31(3), 49-53.]

Zhao L, Ding JC, Ma XF (2011) Principle and Application of Satellite Navigation. Northwestern Polytechnical University Press, Xi'an. (in Chinese) [赵琳, 丁继成, 马雪飞 (2011) 卫星导航原理及应用. 西北工业大学出版社, 西安.]

Civil Aviation Administration of China (CAAC) (2018) An notice of ask comment about "provisional regulations for flight management of unmanned aerial vehicle”. [中国民航 局 (2018) 关于征求《无人驾驶航空器飞行管理暂行条例 (征求意见稿)》意见的通知. http://www.caac.gov. cn/HDJL/YJZJ/201801/t20180126_48853.html. (accessed on 2018-01-26)]

Zhu H, Li YH, Xu ZF, Wang H, Li BG (2001) Characteristics and affinity of the flora of Xishuangbanna, SW China. Guihaia, 21, 127-136. (in Chinese with English abstract) [朱华, 李延辉, 许再富, 王洪, 李宝贵 (2001) 西双版纳植物区 系的特点与亲缘. 广西植物, 21, 127-136.]

(特邀责任编委：郭庆华 责任编辑：问文杰)

\section{附录 Supplementary Material}

附录1 当地森林主要群落类型外貌示意

Appendix 1 Physiognomy of main communities in local forest http://www.biodiversity-science.net/fileup/PDF/2018039-1.pdf 